\title{
Macroscopic Ensembles of Aligned Carbon Nanotubes in Bubble Imprints Studied by Polarized Raman Microscopy
}

\author{
Shota Ushiba, ${ }^{1}$ Jordan Hoyt, ${ }^{2,3}$ Kyoko Masui, ${ }^{1}$ Junichiro Kono, ${ }^{4}$ \\ Satoshi Kawata, ${ }^{1}$ and Satoru Shoji ${ }^{5}$ \\ ${ }^{1}$ Department of Applied Physics, Osaka University, 2-1 Yamadaoka, Suita, Osaka 565-0871, Japan \\ ${ }^{2}$ Department of Mechanical Engineering, University of Tulsa, Tulsa, OK 74104, USA \\ ${ }^{3}$ NanoJapan Program, Department of Electrical and Computer Engineering, Rice University, Houston, TX 77005, USA \\ ${ }^{4}$ Department of Electrical and Computer Engineering, Department of Physics and Astronomy, \\ and Department of Materials Science and NanoEngineering, Rice University, Houston, TX 77005, USA \\ ${ }^{5}$ Department of Engineering Science, The University of Electro-Communications, 1-5-1 Chofugaoka, Chofu, Tokyo 182-8585, Japan
}

Correspondence should be addressed to Satoru Shoji; satoru.shoji@uec.ac.jp

Received 25 April 2014; Accepted 3 June 2014; Published 4 September 2014

Academic Editor: Shota Kuwahara

Copyright (C) 2014 Shota Ushiba et al. This is an open access article distributed under the Creative Commons Attribution License, which permits unrestricted use, distribution, and reproduction in any medium, provided the original work is properly cited.

We study the alignment of single-wall carbon nanotubes (SWCNTs) in bubble imprints through polarized Raman microscopy. A hemispherical bubble containing SWCNTs is pressed against a glass substrate, resulting in an imprint of the bubble membrane with a coffee ring on the substrate. We find that macroscopic ensembles of aligned SWCNTs are obtained in the imprints, in which there are three patterns of orientations: (i) azimuthal alignment on the coffee ring, (ii) radial alignment at the edge of the membrane, and (iii) random orientation at the center of the membrane. We also find that the alignment of SWCNTs in the imprints can be manipulated by spinning bubbles. The orientation of SWCNTs on the coffee ring is directed radially, which is orthogonal to the case of unspun bubbles. This approach enables one to align SWCNTs in large quantities and in a short time, potentially opening up a wide range of CNT-based electronic and optical applications.

\section{Introduction}

Single-wall carbon nanotubes (SWCNTs) consist of onedimensional (1D) graphitic crystalline nanostructures of a rolled-up single layer of graphene formed into hollow cylinder shapes, of which the typical diameter and length are $\sim 1 \mathrm{~nm}$ and $\sim 1000 \mathrm{~nm}$, respectively. SWCNTs have generated great interest due to their extremely high aspect ratios as well as their remarkable mechanical [1], electrical [2], thermal [3], and optical [4] properties. The unique 1D nanostructure leads to the anisotropic properties of SWCNTs, and thus macroscopically aligned SWCNT ensembles can harness these anisotropic properties. The aligned SWCNTs have indeed opened up a wide range of applications such as photodetectors $[5,6]$, polarizers $[7,8]$, actuators [9], and nanocomposites $[10,11]$.

The macroscopic alignment of SWCNTs has been achieved through in situ growth $[12,13]$, mechanical stretching [7, 14], and electric [15] or magnetic [16] field induced alignment methods. Some progress has also been made recently with the macroscopic alignment of SWCNTs by using bubbles $[17,18]$. This method enables one to deposit aligned SWCNTs on large areas and to transfer SWCNTs to different substrates such as glass substrate or flexible plastic sheets.

Here, we also make use of bubbles and investigate the alignment of SWCNTs in bubble imprints. Polarized Raman microscopy was employed to analyze the alignment of SWCNTs. There are several advantages of polarized Raman microscopy over molecular orientation analysis. One of the advantages is that the distribution of SWCNTs, excluding other molecules such as surfactant molecules, can be analyzed in this method. Further, Raman analysis enables one to analyze a relatively large area in a short time, compared to other molecular orientation analysis methods 


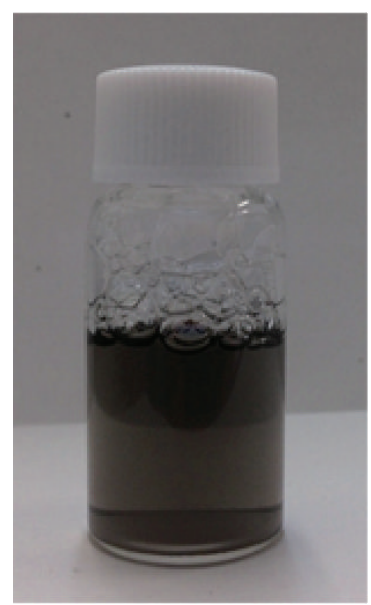

(a)

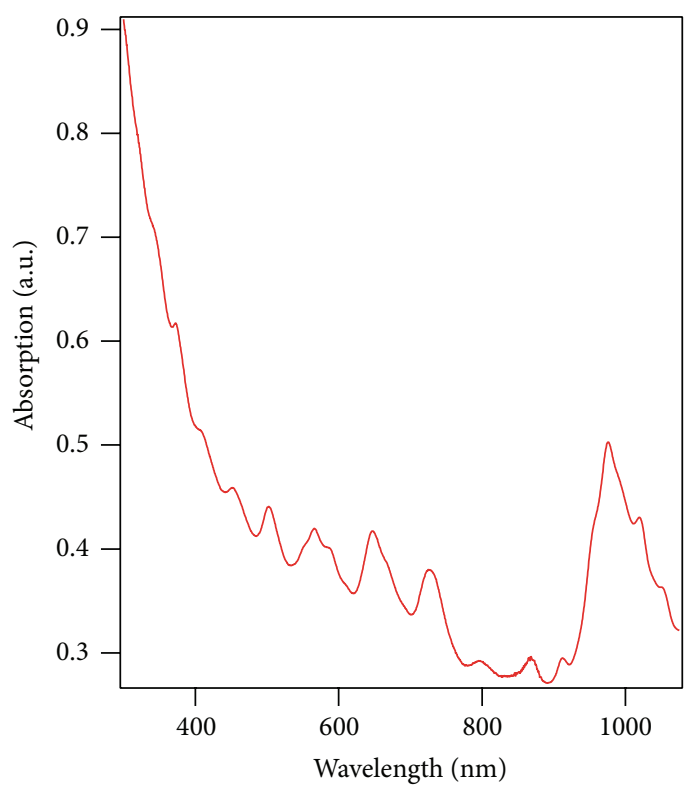

(b)

FIGURE 1: (a) A photograph of SWCNT-dispersed suspension. (b) An absorption spectrum of the SWCNT-dispersed suspension.

such as AFM. Polarized Raman microscopy can also attain a three-dimensional analysis of molecular orientation [19]. In addition, polarized Raman microscopy makes it possible to analyze molecular orientations inside structures besides sample surface $[20,21]$. We thus make use of polarized Raman microscopy for investigating the alignment of SWCNTs in bubble imprints. Furthermore, we also found that the alignment of SWCNTs in the imprints can be manipulated by spinning bubbles during the substrate transfer process.

\section{Experimental}

2.1. Materials. SWCNTs, synthesized by the high-pressure carbon monoxide (HiPco) process at Rice University, were used in this study. The average diameter is $0.97 \mathrm{~nm}$ and the length is about $500-700 \mathrm{~nm}$. $1 \mathrm{wt} \%$ of SWCNTs was loaded into water with $0.3 \mathrm{wt} \%$ of surfactant of sodium dodecylbenzenesulfonate (Aldrich). SWCNTs were subsequently dispersed by ultrasonication for 1 hour. Large aggregated SWCNTs were removed by ultracentrifugation for $10 \mathrm{~min}$ at $173,600 \mathrm{~g}$.

\subsection{The Alignment Study through Polarized Raman Micros-} copy. Polarized Raman microscopy experiments were carried out using a Raman microscope (Raman-11, Nanophoton Corp.) for investigating the orientation direction and degree of alignment of SWCNTs in the bubble imprint. The excitation laser beam, with a wavelength of $785 \mathrm{~nm}$, was linearly polarized after passing through a polarizer and focused onto bubble imprints through an objective lens (100x, NA 0.9 , Nikon). The polarization direction was rotated with a half-wave plate placed between the objective lens and the polarizer. Raman scattering was collected with the same objective lens, and the polarization was rotated to be the same direction as that of the incident light after passing through the half-wave plate. The laser power and exposure time were $4.32 \mathrm{~mW}$ and $5 \mathrm{~s}$, respectively. The polarized Raman intensity depends on the polarization angle, $\theta$, of the Raman excitation laser beam, and the angular dependence of the Raman peak intensity, $I(\theta)$, is fitted with the following function $[22,23]$ :

$$
\begin{aligned}
I(\theta) \propto & \left(\frac{6}{7} \cos ^{2} \theta-\frac{2}{7}\right) \cdot S_{1}+\left(\cos ^{4} \theta-\frac{6}{7} \cos ^{2} \theta+\frac{3}{35}\right) \\
& \cdot S_{2}+\frac{1}{5}
\end{aligned}
$$

where $S_{1}$ and $S_{2}$ are the fitting parameters. $S_{1}$ and $S_{2}$ are the average values of the Legendre polynomials of degrees 2 and 4 and also express the second- and fourth-order orientation parameters, respectively. Both $S_{1}$ and $S_{2}$ equal 1 for perfect alignment and 0 for complete random orientations. Since $S_{1}$ is also known as the nematic order parameter $S$, we use $S$ (or $S_{1}$ ) to represent the strength of the alignment of SWCNTs in this study.

\section{Results}

Figure 1(a) shows a photo of the SWCNT-suspension prepared in this study. To see the dispersion quality of SWCNTs in the solution, we took an absorption spectrum (Figure 1(b)). There are several sharp absorption peaks attributed to interband excitonic transitions in SWCNTs. The appearance of these intrinsic absorption peaks indicates that SWCNTs are well dispersed in the solution.

Bubbles were prepared from the suspension, as illustrated in Figure 2(a). A hemispherical bubble was created at room temperature on a glass substrate (Matsunami micro cover glass, $22 \times 40 \mathrm{~mm}$ ) using a plastic pipette (Figure $2(\mathrm{~b})$ ). The 


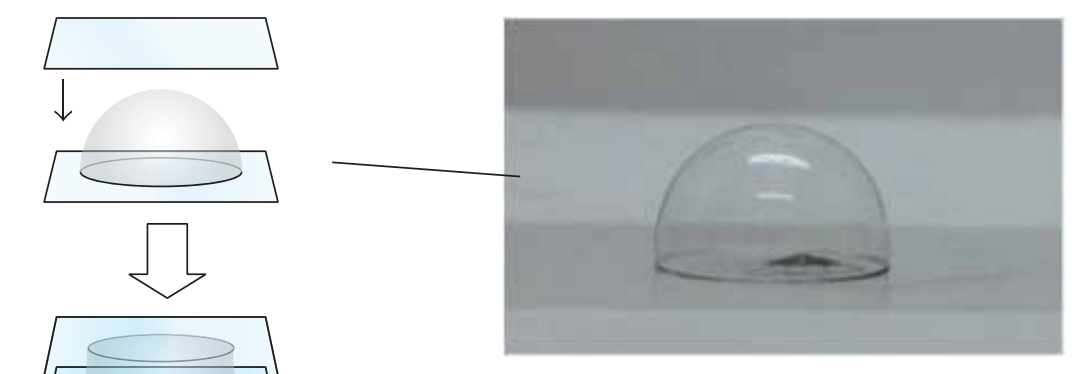

(b)

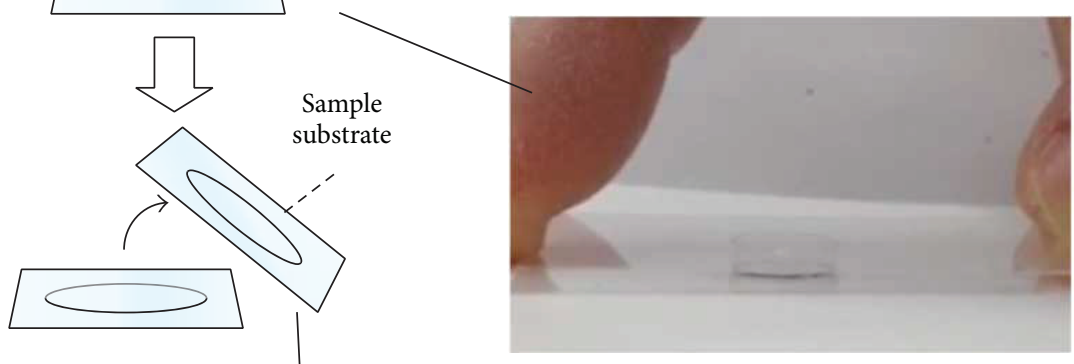

(a)

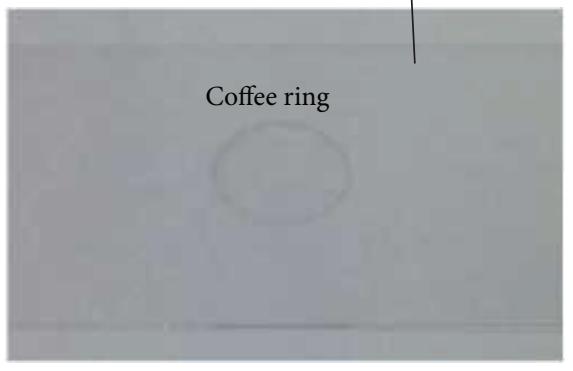

(d) (c)

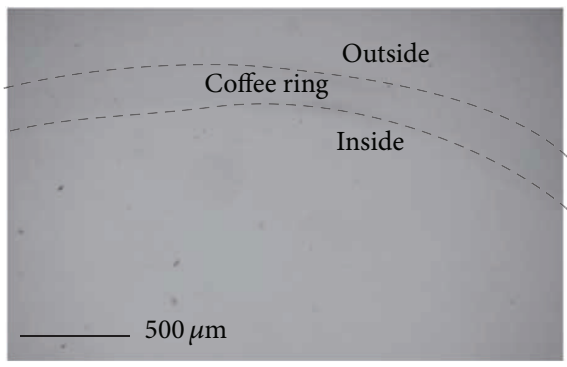

(e)

FIgURE 2: (a) Procedure of bubble printing to a glass substrate. (b) A photograph of a hemispherical bubble containing SWCNTs on a glass substrate. (c) A photograph of a bubble under compression. (d) A photograph of a bubble imprint. (e) A bright field image of a bubble imprint.

typical diameter was around $15-20 \mathrm{~mm}$, and the thickness of the bubble membrane was around $1 \mu \mathrm{m}$. The membrane was composed of three layers: the inner and outer surfaces of these bubbles were made up of surfactant, and the middle layer was composed of SWCNTs and water [18]. The bubble was pressed against another glass substrate (Matsunami micro cover glass, $22 \times 40 \mathrm{~mm}$ ) until the bubble popped (Figure $2(\mathrm{c})$ ). The typical lifetime of bubbles was 5-20 s. The bubble was imprinted on to the flat surface with a coffee ring with a width of $\sim 100 \mu \mathrm{m}$ (Figures 2(d) and 2(e)). The obtained membrane imprint was used for testing.

Figures 3(a) and 3(b) present a typical Raman spectrum taken from a bubble imprint. The result shows multiple peaks in the range of 200 to $270 \mathrm{~cm}^{-1}$ and a pronounced peak at $1585 \mathrm{~cm}^{-1}$, assigned to the RBM and G-band of SWCNTs, respectively. This result gives a clear indication that SWCNTs are contained in bubbles and also transferred to the substrate through the printing process.

The alignment of SWCNTs in the bubble membrane was investigated by measuring the polarization-angular dependence of the $G$-band intensity, $I_{G}(\theta) . \theta$ is defined as an angle between the polarization angle of the Raman excitation laser beam and $x$-axis (Figure 4(a)). Figures $4(\mathrm{~b})-4(\mathrm{e})$ show $I_{G}(\theta)$ at four different positions signed in Figure 4(a): at " $b$ " the top edge of the membrane (Figure 4(b)), at " $c$ " the right edge of the membrane (Figure 4(c)), at " $\mathrm{d}$ " the center of the membrane (Figure $4(\mathrm{~d})$ ), and at "e" on coffee ring at the right side (Figure 4(e)). The plots in Figures 4(c) and 4(d) show that the $G$-band intensity becomes largest when the polarization is parallel to the radial direction, while the intensity becomes smallest when the polarization is perpendicular. From the fitting curve, the nematic order parameter $S$ is calculated to be 0.23 and 0.19 at the positions "b" and "c", respectively. These results clearly indicate that SWCNTs embedded in the membrane are oriented toward the center of the imprint. On the contrary, the plot in Figure 4(d) corresponding to the near center shows no specific angular dependence, as $S$ is obtained as 0.07 . This result indicates that SWCNTs are randomly oriented at the center. It is worth noting that the alignment on the coffee ring (Figure 4(e)) differs from that of the inside (Figure 4(c)). The G-band intensity becomes largest when the polarization is parallel to the boundary, while the intensity becomes smallest when the polarization is perpendicular. The result indicates that SWCNTs on the coffee ring are oriented along the coffee ring, namely, azimuthally aligned. $S$ at the position " $\mathrm{e}$ " on the coffee ring is calculated to be 0.52 . 


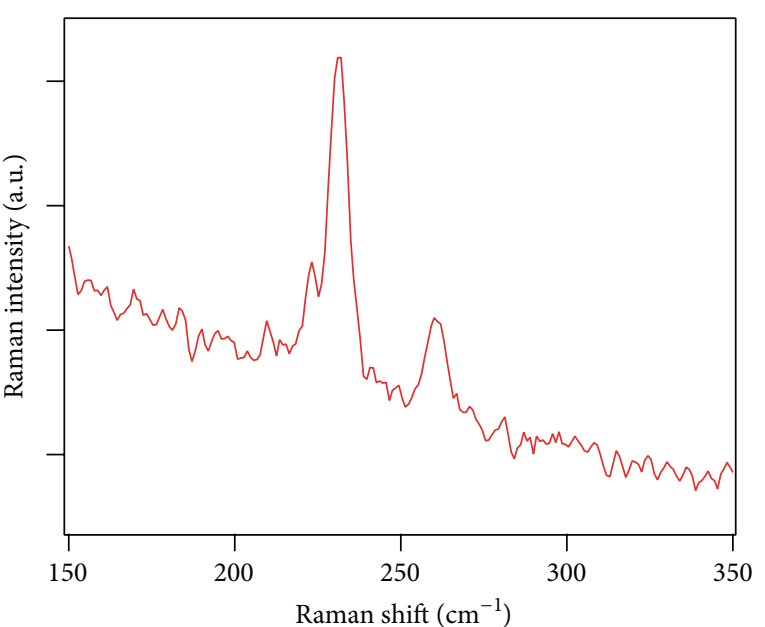

(a)

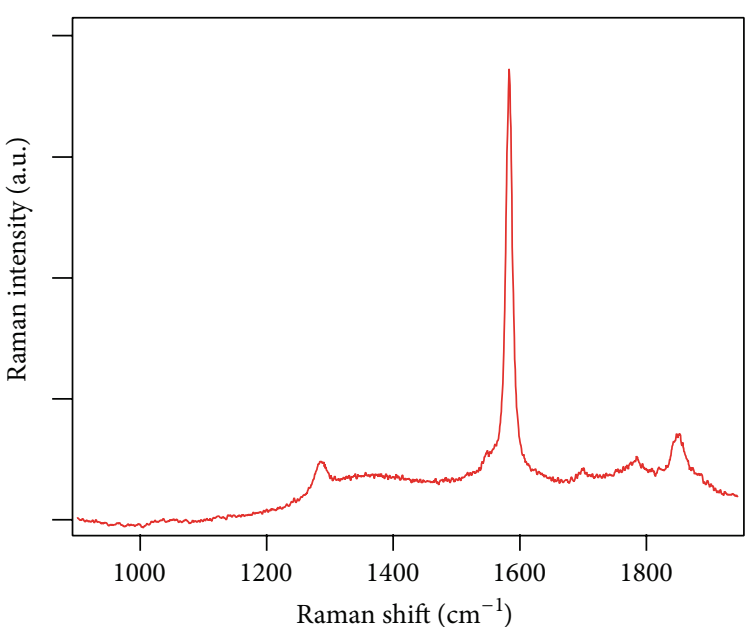

(b)

FIGURE 3: Raman spectrum taken from a bubble imprint in RBM region (a) and D-band and G-band region (b).

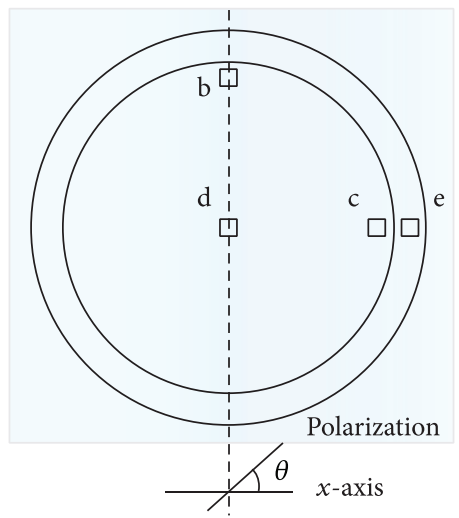

(a)

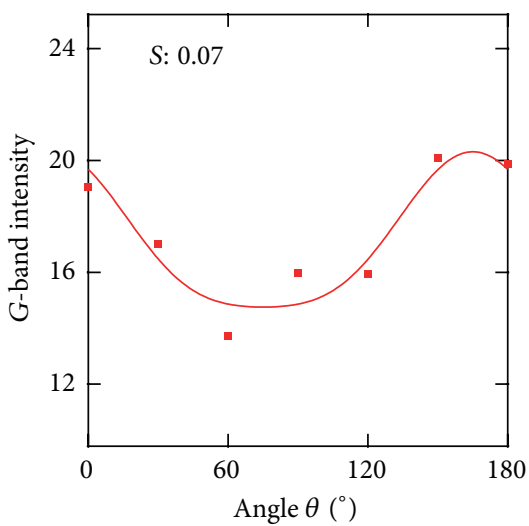

(d)

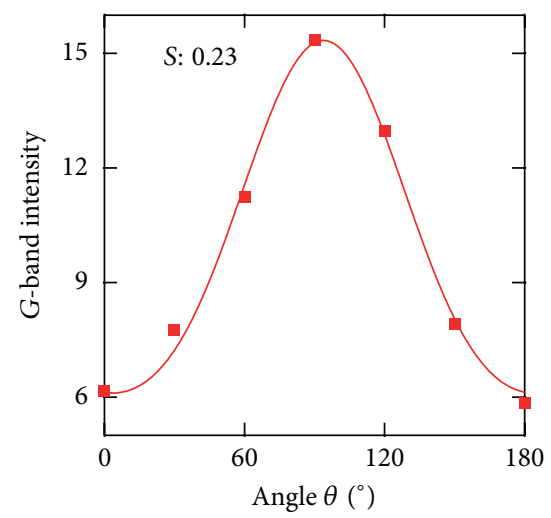

(b)

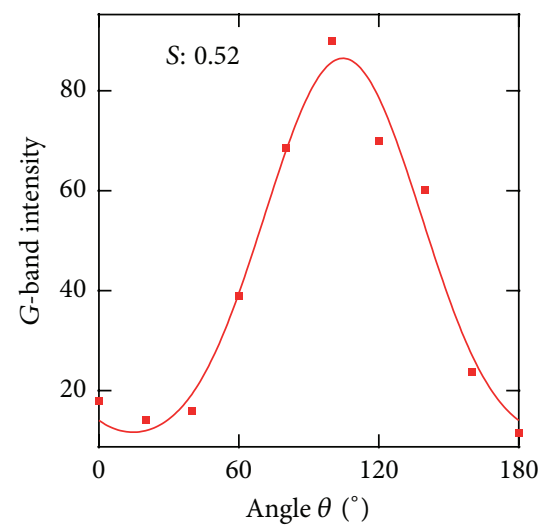

(e)

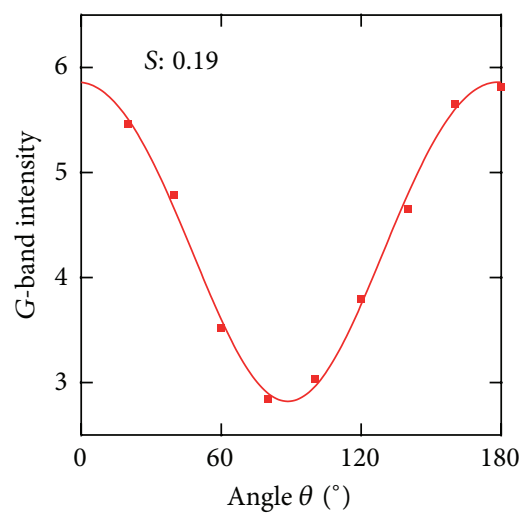

(c)

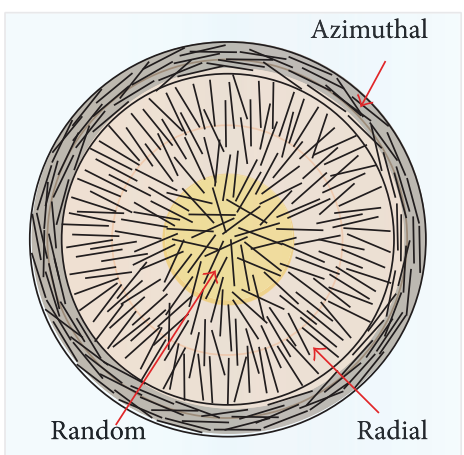

(f)

Figure 4: (a) Schematic of a bubble imprint representing measurement areas with polarized Raman microscopy. $\theta$ is defined as an angle between the laser polarization and $x$-axis. Polarization angular dependence taken from (b) the top edge of the bubble membrane, (c) the right edge of the bubble membrane, (d) the center of the membrane, and (e) in the coffee ring. $S$ is defined as the nematic order parameters. (f) Illustration showing the orientation of SWCNTs in a bubble imprint. 


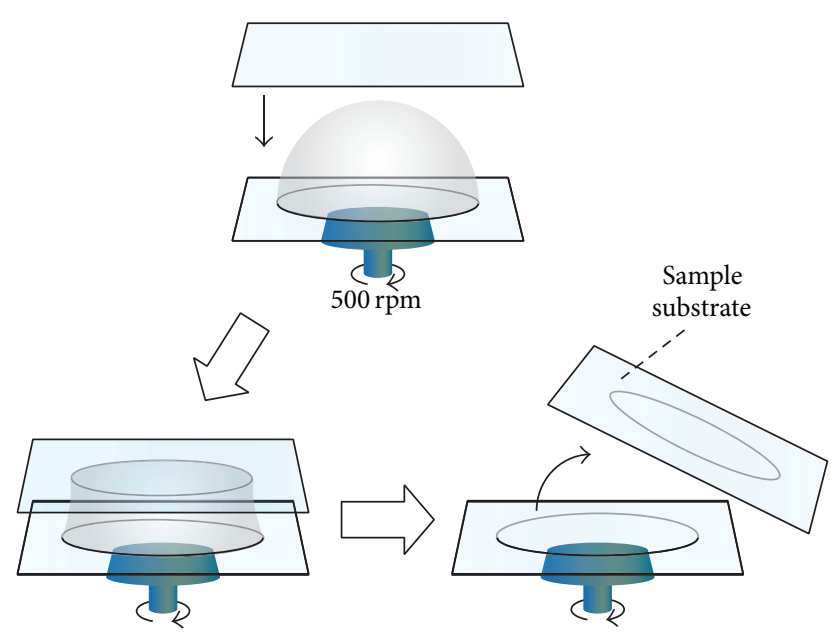

(a)

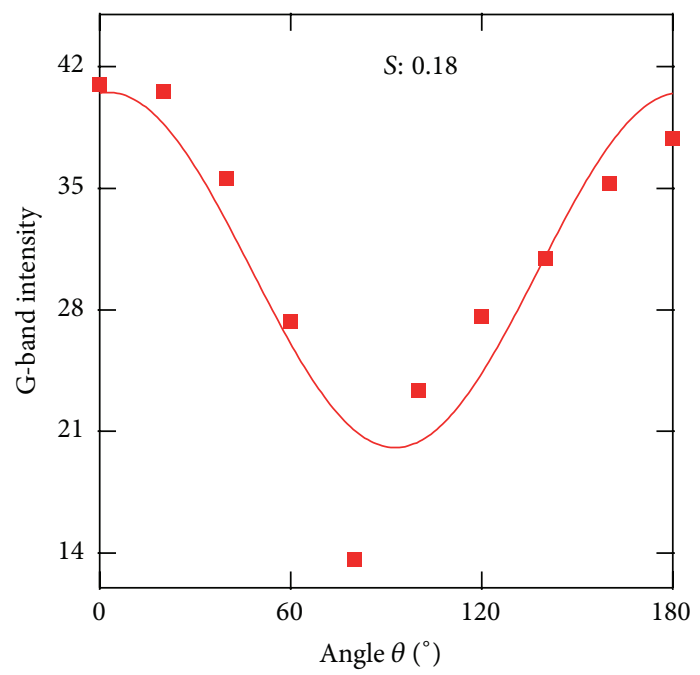

(c)

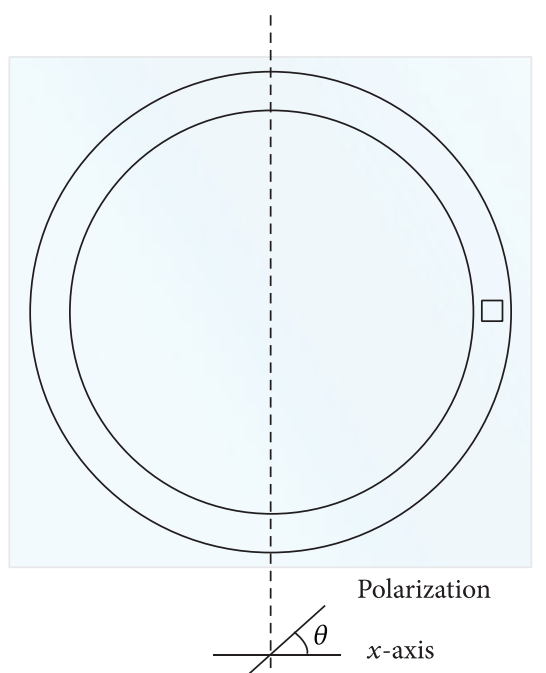

(b)

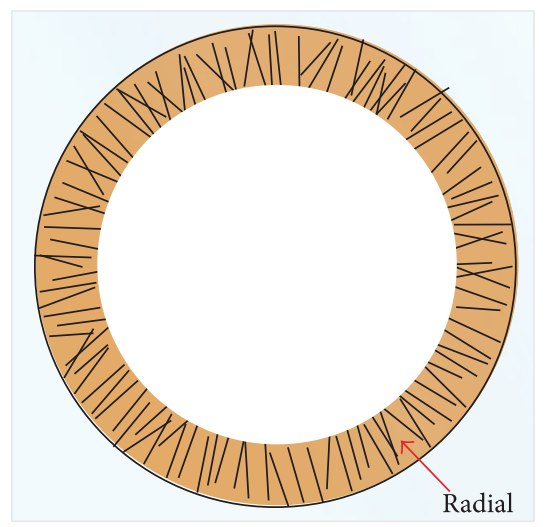

(d)

Figure 5: (a) Schematic of a spun bubble imprint representing measurement areas with polarized Raman microscopy. $\theta$ is defined as an angle between the laser polarization and $x$-axis. (b) Polarization angular dependence taken from the coffee ring at the right boundary. (c) Illustration showing the orientation of SWCNTs in a spun bubble imprint.

This means that the SWCNTs on the coffee ring are more strongly aligned compared to the SWCNTs in the inside of the coffee ring. On the basis of the obtained results in Figures 4(b)-4(e), the alignment of SWCNTs in the imprint is depicted in Figure 4(f). There are three patterns of alignment of SWCNTs: (i) radial alignment at the inside of the coffee ring, (ii) azimuthal alignment on the coffee ring, and (iii) random orientation at the center.

We demonstrated the same experiment with spinning bubbles. The printing process of a spun bubble is shown in Figure 5(a). A bubble was created on a glass substrate, and subsequently the glass substrate was spun at $500 \mathrm{rpm}$ using a spin coater. The speed of $500 \mathrm{rpm}$ was the highest speed that we could perform the experiments at due to bubble instability at higher rotational speeds. The spun bubble was pressed by another glass substrate that was held until the bubble popped, just as was done for an unspun bubble. The alignment of SWCNTs in the spun bubble was investigated with the same polarized Raman microscopy setup described above. $\theta$ is defined as an angle between the laser polarization and $x$-axis (Figure 5(b)). Figure 5(c) shows $I_{G}(\theta)$ taken from the right side of the coffee ring. In the plot, the $G$-band intensity becomes largest when the polarization is parallel to the boundary $\left(\theta \sim 0^{\circ}\right)$, while the intensity becomes smallest when the polarization is perpendicular $\left(\theta \sim \pm 90^{\circ}\right)$. This means that SWCNTs on the coffee ring were radially aligned, as illustrated in Figure 5(d). The result differs from that of the unspun bubble that exhibits azimuthal alignment on the coffee ring. $S$ for the coffee ring was calculated to be 0.18 . This value is smaller compared to unspun bubbles $(S \sim 0.52)$. On the other hand, in the case of a spun bubble, no $G$-band Raman signal was detected at the inside of the coffee 
ring. This result implies that there are no or few SWCNTs at the inside, probably due to the centrifugal force that makes SWCNTs go away from the center of the bubble during spinning.

\section{Discussion}

Here, we separate alignment mechanisms for inside the coffee ring and on the coffee ring. For the alignment inside of the coffee ring, the alignment is induced by water runoff. After bubbles are created, water runs down from the top of the bubbles due to the drainage [18]. This water flow aligns SWCNTs toward the water-flow direction, resulting in the radial orientation inside the coffee ring. By contrast, at the top, SWCNTs do not flow in specific directions, resulting in the random orientation at the center of the bubble imprints.

For the coffee ring, the alignment occurs during evaporation of a coffee ring shaped droplet. There is a successful report that shows, in a microsized hemicylindrical droplet, SWCNTs were aligned along the long axis of the droplet during evaporation [24]. This alignment was induced by an internal hydrodynamic flow of a drying hemicylindrical droplet that carries nanotubes to the interface. The same alignment mechanism should also work in ring shaped hemicylindrical droplets in our study, thereby leading to the azimuthal alignment on the coffee ring.

However, as observed in our experimental results, the alignment on the coffee ring created from a spun bubble differed from that of an unspun bubble. We presume that the alignment does not occur during evaporation of a coffee ring shaped droplet in the spun bubble case because the coffee ring shaped droplet was blurry after bubble printing, compared to the unspun bubble case. We think that the realignment does not occur during the evaporation because of quick evaporation resulting from thinly spread out liquid. Instead, the alignment in the bubble membrane, that is, radial alignment, is imprinted to the substrate. The different alignment mechanism from unspun bubbles possibly results from the centrifugal force during spinning process. As a bubble is spun, the bubble is slightly expanded and flattened due to centrifugal force, leading to further thinning of the bubble membrane. Therefore, the coffee ring shaped droplet created after bubble imprints was much shorter in height and spread out when compared to the unspun bubble case.

The overall results are statistically reproducible, and the reproducibility of the results in the given condition is $\sim 70 \%$ for unspun bubbles and $\sim 40 \%$ for spun bubbles. The reproducibility could be improved by optimizing experimental conditions. We think the following two parameters are the most important to improve the reproducibility: (1) timing of the bubble printing after bubble creation and (2) concentration of both SWCNTs and surfactants. It is discussed in a previous report of Tang et al. [18] that if the bubble film is transferred to a substrate at too early of a stage, that is, right after bubble creation, one might obtain a random SWCNT distribution. This is because the alignment relies on the self-organization of SWCNTs in bubble membrane and so SWCNTs are not fully aligned at the early stage. Tang et al. [18] also noted that the concentration of SWCNT and surfactants is a critical parameter for the alignment in the bubble printing method, because SWCNTs cannot selforganize their alignment in bubble membranes under low concentration of SWCNTs and/or high concentration of surfactants. We thus presume that optimizing these parameters will lead to an improvement of the reproducibility and the strength of the alignment.

\section{Conclusion}

We have studied the alignment of SWCNTs in bubble imprints through polarized Raman microcopy. There are three patterns of alignment of SWCNTs in the imprints: radial, azimuthal, and random orientations, which are found at the near boundary, on the coffee ring, and the center, respectively. We suggest that the alignment mechanism involves two factors: water molecule runoff, and evaporation. We have also found that the alignment of SWCNTs in the imprints can be manipulated by spinning bubbles. The bubble spun at $500 \mathrm{rpm}$ exhibits radial alignment of SWCNTs on the coffee ring, which is rotated by 90 degrees, compared to unspun bubbles. Although the rotational speed dependence has not been examined, this result implies a possibility to manipulate the alignment in bubble imprints. The bubble imprint method provides macroscopic ensembles of aligned SWCNTs, whose nematic order parameters, $S$, reach 0.5 . Although the strength of the alignment of SWCNTs obtained by the bubble printing method is weaker than or comparable to other methods such as in situ growth methods [12, 13] and mechanical stretching methods [7, 14], our bubble imprints would be enough to exhibit anisotropic properties including electrical and thermal conductivity [16]. The bubble printing method, thus, leads to a wide range of applications that include electronic $[5,6]$ and optical devices $[7,8]$.

\section{Conflict of Interests}

The authors declare that there is no conflict of interests regarding the publication of this paper.

\section{Acknowledgments}

This research is supported by The Canon Foundation and KAKENHI Grant-in-Aid for Young Scientists (nos. 21686010 and 19810012), MEXT, Japan. Junichiro Kono acknowledges financial support from the US National Science Foundation (through Grant no. OISE-0968405), the Department of Energy (through Grant no. DE-FG02-06ER46308), and the Robert A. Welch Foundation (through Grant no. C-1509). Shota Ushiba thanks Dr. Susan Skelton for helping to write this paper. 


\section{References}

[1] A. Krishnan, E. Dujardin, T. W. Ebbesen, P. N. Yianilos, and M. M. J. Treacy, "Young's modulus of single-walled nanotubes," Physical Review B, vol. 58, no. 20, pp. 14013-14019, 1998.

[2] S. J. Tans, M. H. Devoret, H. Dai et al., "Individual single-wall carbon nanotubes as quantum wires," Nature, vol. 386, no. 3, pp. 474-477, 1997.

[3] J. Hone, B. Batlogg, Z. Benes, A. T. Johnson, and J. E. Fischer, "Quantized phonon spectrum of single-wall carbon nanotubes," Science, vol. 289, no. 5485, pp. 1730-1733, 2000.

[4] S. M. Bachilo, M. S. Strano, C. Kittrell, R. H. Hauge, R. E. Smalley, and R. B. Weisman, "Structure-assigned optical spectra of single-walled carbon nanotubes," Science, vol. 298, no. 5602, pp. 2361-2366, 2002.

[5] W. He and T. Ren, "Basilar membrane vibration is not involved in the reverse propagation of otoacoustic emissions," Scientific Reports, vol. 3, article 1874, 2013.

[6] X. He, X. Wang, S. Nanot et al., "Photothermoelectric p-n junction photodetector with intrinsic broadband polarimetry based on macroscopic carbon nanotube films," ACS Nano, vol. 7, no. 8, pp. 7271-7277, 2013.

[7] S. Shoji, H. Suzuki, R. P. Zaccaria, Z. Sekkat, and S. Kawata, "Optical polarizer made of uniaxially aligned short singlewall carbon nanotubes embedded in a polymer film," Physical Review B-Condensed Matter and Materials Physics, vol. 77, no. 15, Article ID 153407, 2008.

[8] L. Ren, C. L. Pint, L. G. Booshehri et al., "Carbon nanotube terahertz polarizer," Nano Letters, vol. 9, no. 7, pp. 2610-2613, 2009.

[9] S. V. Ahir and E. M. Terentjev, "Photomechanical actuation in polymer-nanotube composites," Nature Materials, vol. 4, no. 6, pp. 491-495, 2005.

[10] L. Ci, J. Suhr, V. Pushparaj, X. Zhang, and P. M. Ajayan, "Continuous carbon nanotube reinforced composites," Nano Letters, vol. 8, no. 9, pp. 2762-2766, 2008.

[11] S. Ushiba, S. Shoji, K. Masui, P. Kuray, J. Kono, and S. Kawata, "3D microfabrication of single-wall carbon nanotube/polymer composites by two-photon polymerization lithography," Carbon, vol. 59, pp. 283-288, 2013.

[12] L. Ding, D. Yuan, and J. Liu, "Growth of high-density parallel arrays of long single-walled carbon nanotubes on quartz substrates," Journal of the American Chemical Society, vol. 130, no. 16, pp. 5428-5429, 2008.

[13] W. Z. Li, S. S. Xie, L. X. Qian et al., "Large-scale synthesis of aligned carbon nanotubes," Science, vol. 274, no. 5293, pp. 17011703, 1996.

[14] L. Jin, C. Bower, and O. Zhou, "Alignment of carbon nanotubes in a polymer matrix by mechanical stretching," Applied Physics Letters, vol. 73, no. 9, pp. 1197-1199, 1998.

[15] C. Park, J. Wilkinson, S. Banda et al., "Aligned single-wall carbon nanotube polymer composites using an electric field," Journal of Polymer Science B: Polymer Physics, vol. 44, no. 12, pp. 1751-1762, 2006.

[16] J. E. Fischer, W. Zhou, J. Vavro et al., "Magnetically aligned single wall carbon nanotube films: preferred orientation and anisotropic transport properties," Journal of Applied Physics, vol. 93, no. 4, pp. 2157-2163, 2003.

[17] G. Yu, A. Cao, and C. M. Lieber, "Large-area blown bubble films of aligned nanowires and carbon nanotubes," Nature Nanotechnology, vol. 2, no. 6, pp. 372-377, 2007.
[18] G. Tang, X. Zhang, S. Yang, V. Derycke, and J. Benattar, "New confinement method for the formation of highly aligned and densely packed single-walled carbon nanotube monolayers," Small, vol. 6, no. 14, pp. 1488-1491, 2010.

[19] T. Mino, Y. Saito, H. Yoshida, S. Kawata, and P. Verma, "Molecular orientation analysis of organic thin films by z-polarization Raman microscope," Journal of Raman Spectroscopy, vol. 43, no. 12, pp. 2029-2034, 2012.

[20] J. Purvis and D. I. Bower, "A study of molecular orientation in poly(methyl methacrylate) by means of laser-Raman spectroscopy," Polymer, vol. 15, no. 10, pp. 645-654, 1974.

[21] M. Richard-Lacroix and C. Pellerin, "Orientation and structure of single electrospun nanofibers of poly (ethylene terephthalate) by confocal Raman spectroscopy," Macromolecules, vol. 45, no. 4, pp. 1946-1953, 2012.

[22] J. Shaver, A. N. G. Parra-Vasquez, S. Hansel et al., "Alignment dynamics of single-walled carbon nanotubes in pulsed ultrahigh magnetic fields," ACS Nano, vol. 3, no. 1, pp. 131-138, 2009.

[23] T. Liu and S. Kumar, "Quantitative characterization of SWNT orientation by polarized Raman spectroscopy," Chemical Physics Letters, vol. 378, no. 3-4, pp. 257-262, 2003.

[24] R. Sharma, C. Y. Lee, J. H. Choi, K. Chen, and M. S. Strano, "Nanometer positioning, parallel alignment, and placement of single anisotropic nanoparticles using hydrodynamic forces in cylindrical droplets," Nano Letters, vol. 7, no. 9, pp. 2693-2700, 2007. 

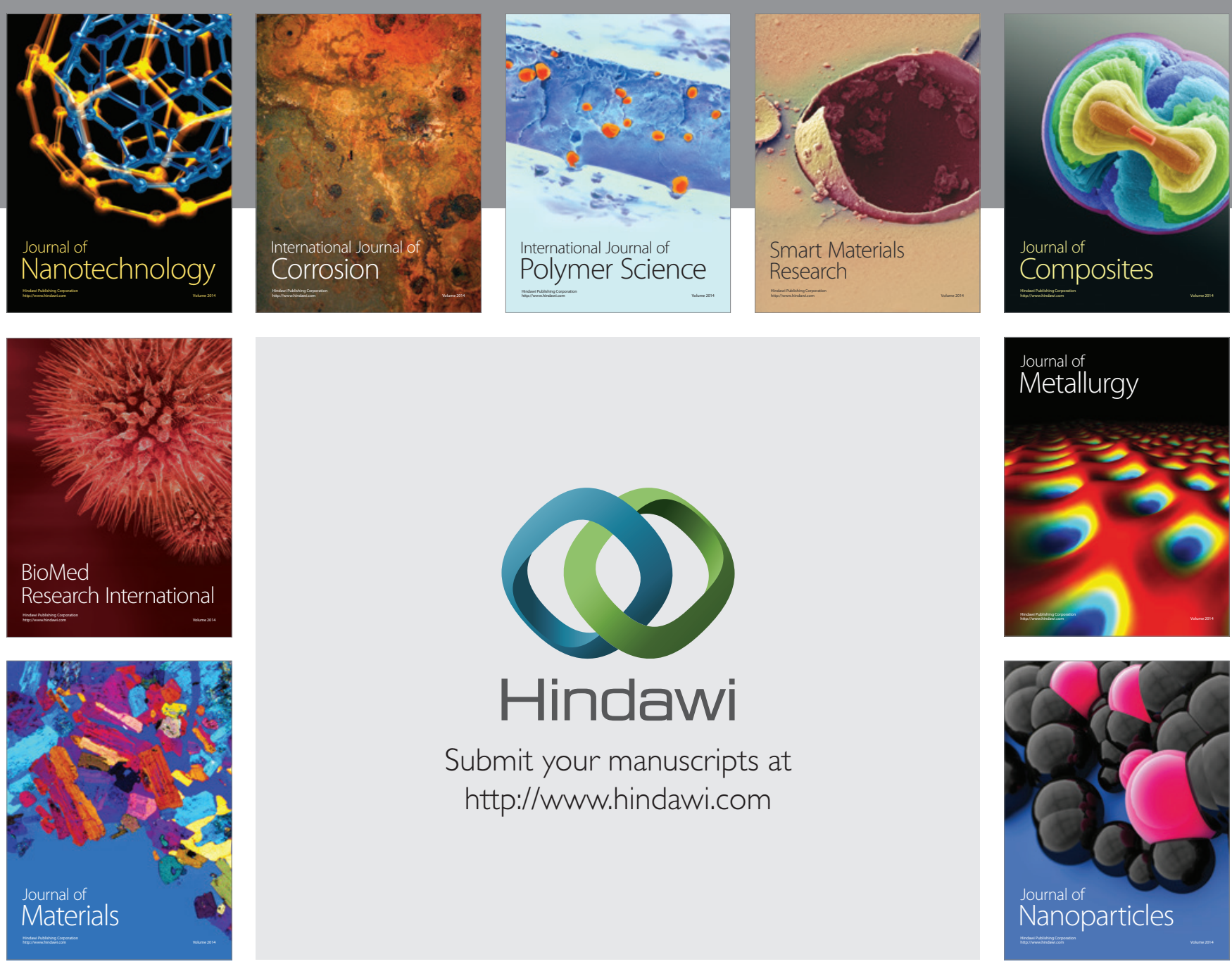

Submit your manuscripts at http://www.hindawi.com
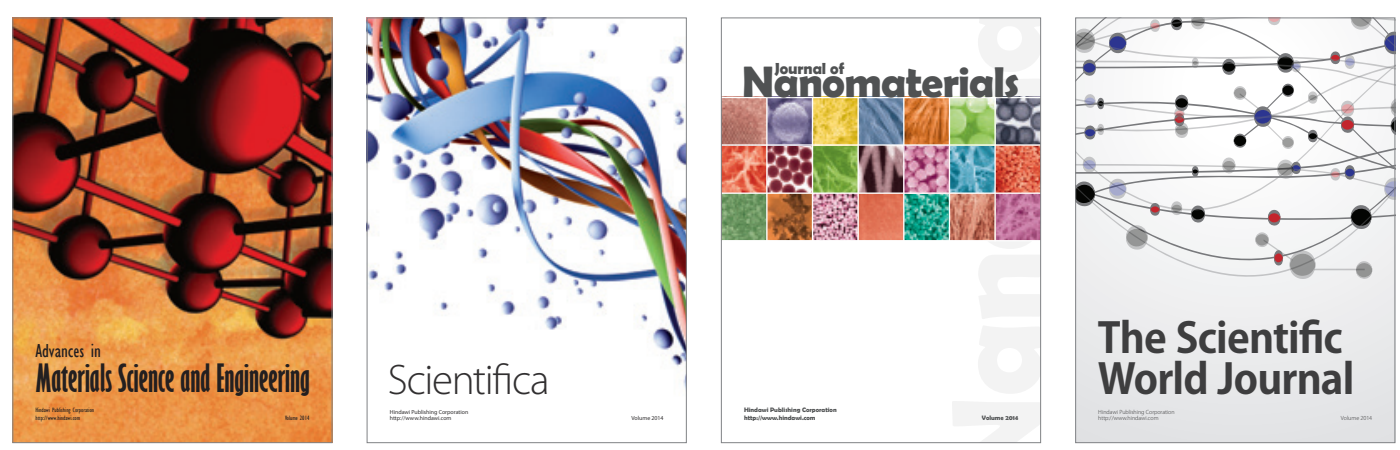

\section{The Scientific World Journal}
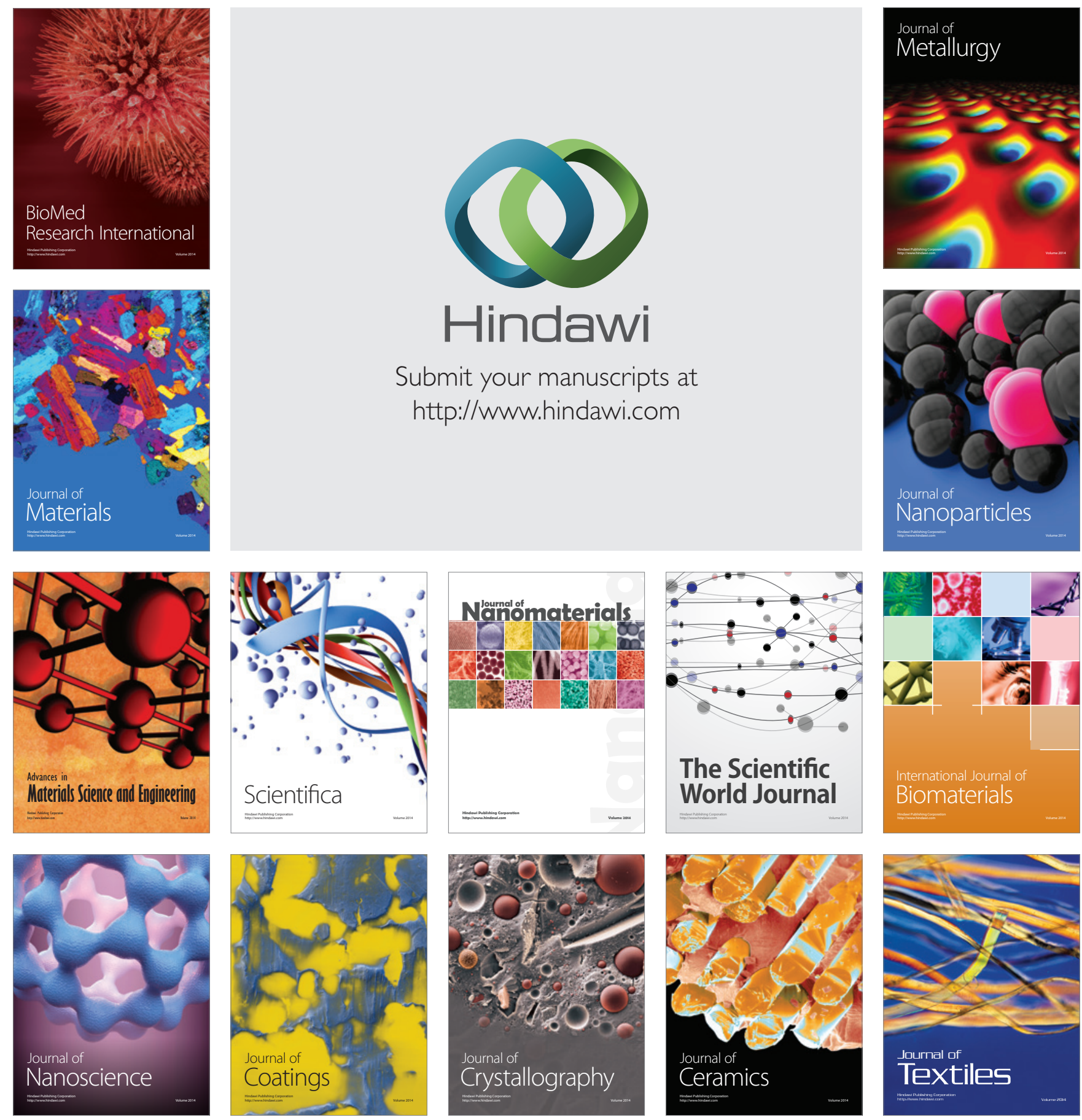